\title{
Artículos
}

\section{La inteligencia competitiva en España: una encuesta sobre su utilización por parte de las empresas exportadoras}

\author{
Por Jorge Postigo
}

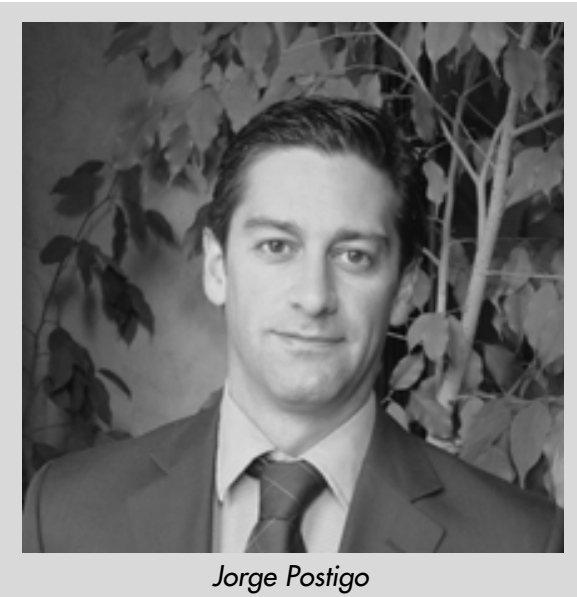

Resumen: El artículo analiza los resultados de una encuesta sobre el uso de la inteligencia competitiva entre empresas exportadoras españolas y explica las características de cuatro sistemas formales actualmente en funcionamiento. Se confirma que la utilización de un sistema formal de seguimiento del entorno competitivo concede a las empresas una ventaja competitiva; y que su implementación está justificada independientemente del tamaño de la empresa. A pesar de ello es evidente que la inteligencia competitiva es una disciplina desconocida en España y que salvo excepciones las empresas no abordan esta tarea de forma sistemática. Para llegar a esta conclusión se ofrece una visión general de las necesidades de información de las empresas exportadoras españolas, del uso que éstas realizan de distintas técnicas de inteligencia, y de los principales obstáculos con que se encuentran para implementar procesos formales.

Palabras clave: Inteligencia competitiva, Exportación, Gestión del conocimiento, Pymes, Pequeñas y medianas empresas.

Title: Competitive intelligence in Spain: a survey of its use by Spanish exporters

Abstract: This article analyses the results of a survey among Spanish exporters, and the experiences of four organisations implementing a formal competitive intelligence programme. The research confirms that a formal approach to competitive intelligence, rather than informal or discontinuous approaches, gives businesses of all sizes competitive advantage, and justifies the value of its implementation by Spanish exporters. However, competitive intelligence as a business discipline remains largely unknown and misunderstood in Spain, and few organisations implement systematic programmes to benefit from the intelligence effort. To reach this conclusion the article offers a view of the information needs of Spanish exporters, the use of different intelligence techniques, and the main obstacles they find when implementing formal intelligence systems.

Keywords: Competitive intelligence, Exporters, Knowledge management, SMEs, Small and medium size enterprises.

Postigo, Jorge. "La inteligencia competitiva en España: una encuesta sobre su utilización por parte de las empresas exportadoras". En: El profesional de la información, v. 10, n. 10, pp.4-11.

\section{Introducción}

El trabajo aquí presentado forma parte de un estudio más amplio titulado "Competitive intelligence in Spain: an investigation into current practices and future possibilities", realizado como parte de un programa MBA (master of business administration) en el Henley Management College. La hipótesis de partida consistía en probar si un proceso formal de recogida, análisis y diseminación de información sobre la competencia puede optimizar el proceso interno de toma de decisiones a través de un mejor entendimiento del entorno competitivo.

El estudio ha sido motivado por la necesidad de comprender los esfuerzos de las empresas exportadoras por transformar una enorme cantidad de información sobre su entorno en inteligencia susceptible de ser aprovechada de forma eficaz en los mercados interna- cionales. En un mundo donde la competencia aumenta de forma continua, la gestión eficaz de la información sobre la competencia es una herramienta de gran valor para la dirección de la compañía. Muchos autores se han referido a la importancia de su utilización estratégica, incluso desde estas páginas (ver EPI, v. 10, n. 5) se han tratado recientemente diversos aspectos de lo que comúnmente se denomina inteligencia competitiva (ic).

Un sistema, función o programa formal de ic es un proceso estructurado y sistemático de obtención, análisis, difusión y protección de la información sobre el entorno competitivo. Puede estar centralizado en una unidad o persona, o descentralizado en varias áreas de la empresa, pero siempre conlleva una coordinación. Los sistemas formales se diferencian de las actividades informales de seguimiento del entorno en que éstas, 
que han existido siempre, se pueden realizar de manera sistemática, pero sin una coordinación y un proceso.

Hay una tercera variante de ic, que podría denominarse como discontinua, cuando el seguimiento del entorno se realiza de forma puntual, es decir, sólo cuando es necesario para la toma de decisiones, sin seguir un procedimiento.

\section{«El estudio ha sido motivado por la necesidad de compren- der los esfuerzos de las empre- sas exportadoras por transfor- mar en inteligencia una enorme cantidad de información sobre su entorno»}

En este artículo no se tratan los conceptos teóricos de la ic, sino que se analiza su práctica en España en todas las vertientes mencionadas. En el momento de realización del informe no existía investigación de campo sobre la materia en este país por lo que, para tener una visión general de la situación fue necesario abordar a un amplio espectro de organizaciones, de tamaños y sectores diversos. Para ello recurrimos a las opiniones de 281 directivos y al estudio de cuatro experiencias reales en la implantación de unidades de inteligencia.

\section{Principales resultados de la encuesta}

a. ¿Hay necesidad de utilizar ic por parte de las empresas?

El 55\% de ellas exporta menos del $30 \%$ de su producción, operando en mercados en los que el cambio tecnológico es elevado. El $60 \%$ observa la entrada de nuevos competidores en su radio de actuación, mientras que el $74 \%$ considera que su principal reto futuro es el acceso a nuevos mercados de exportación (ver gráficos 1 y 2).

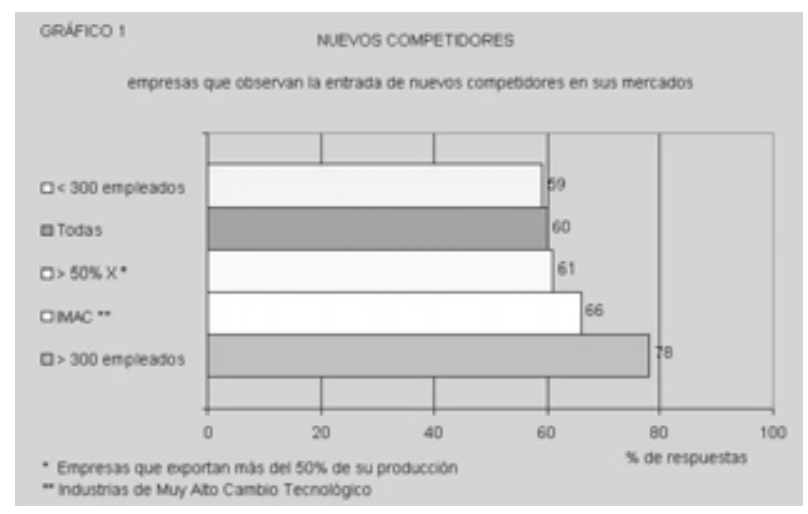

Las empresas españolas, en especial las pymes (pequeñas y medianas empresas), deberán incrementar

\section{Ficha técnica de la encuesta}

Ámbito: Nacional español.

Universo: Directores y responsables de exportación de empresas españolas.

Fuente de datos: Bases de datos del Departamento de Productos Industriales y Servicios de la Oficina Comercial de España en Londres.

Muestra: 716 empresas segmentadas por número de empleados y sector. El índice de respuesta de cuestionarios válidos fue del 39,3\%.

Método de recogida de datos: Mediante cuestionario con catorce preguntas enviado por correo electrónico, de forma que los encuestados recibían un mensaje con el formulario adjunto. A mitad del periodo dedicado al trabajo de campo se envió un recordatorio.

Tratamiento de los datos: Las respuestas se introdujeron en una base de datos creada en Access, diseñada al efecto. El trabajo de campo fue realizado por Jorge Postigo, incluyendo el diseño y tabulación de cuestionarios y gráficos.

Fechas de realización de la encuesta: Entre julio y septiembre de 2000.

significativamente sus exportaciones en los próximos años. Sin embargo, cuanto mayor sea su actividad exportadora, y el número de nuevos competidores aumente, más grande será su necesidad de inteligencia. Ante esta perspectiva, ¿están las empresas españolas haciendo uso de la ic?

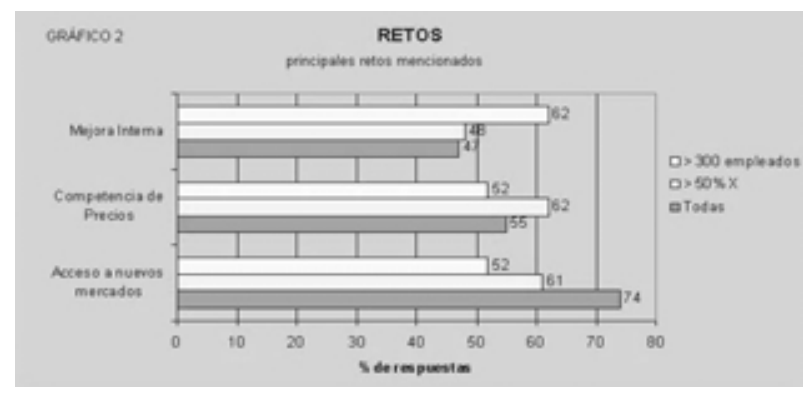

b. Conocimiento del significado de ic.

A pesar del creciente interés por esta forma de actuar en España, sólo el 33\% de los encuestados afirma conocer su significado (gráfico 3). Existen tres factores que explican su escaso conocimiento. En primer lugar hay un problema de definición: algunas empresas realizan actividades de ic pero no utilizan este término para referirse a ellas. El uso de la palabra "inteligencia" puede tener connotaciones negativas asociadas al espionaje industrial, por lo que en algunos casos se opta por utilizar otras denominaciones. 
En segundo lugar, debido a que la ic impregna todas las áreas de la organización, a menudo se dificulta su distinción de otras disciplinas como el marketing, la estrategia o la investigación de mercados.

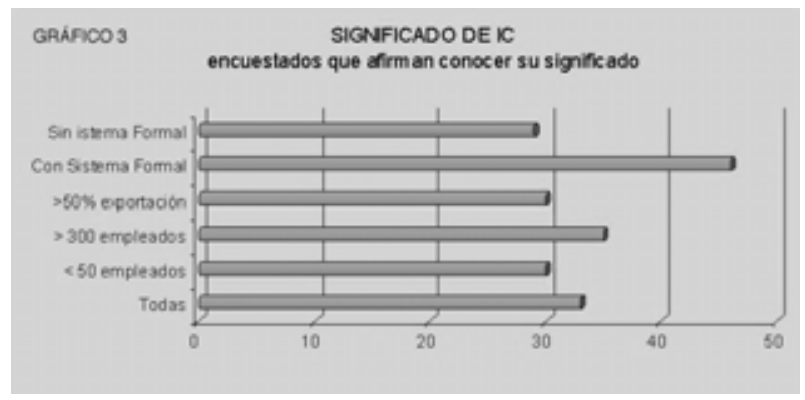

Por último, como proceso formal, es una disciplina relativamente nueva en un país donde predominan las pymes, que encuentran por lo general más dificultades que las organizaciones grandes para implementar los procesos sistemáticos y estructurados que la ic exige. Es de suponer que esta situación no es exclusiva de España, sino que se produce en otros países de nuestro entorno con estructuras macroeconómicas similares.

\section{«Las empresas grandes que han alcanzado cuotas acepta- bles de exportación están ma- yoritariamente interesadas en la mejora interna de sus proce- sos a través de nuevas tecno- logías»}

El gráfico 2 demuestra que las empresas grandes que han alcanzado cuotas aceptables de exportación están mayoritariamente interesadas en la mejora interna de sus procesos a través de nuevas tecnologías. Esto indicaría su disposición a incorporar técnicas modernas de gestión para intensificar su competitividad, lo que a su vez supone una oportunidad para la adopción de prácticas de ic. Su uso por parte de las empresas de mayor tamaño podría tener un efecto dominó sobre las más pequeñas, que lo verían como un ejemplo a seguir.

\section{c. La ic como prioridad en la empresa.}

Para el $71 \%$ de los directivos, cuando se trata de tomar decisiones estratégicas un buen conocimiento de las actividades de la competencia es al menos tan importante como el que se obtiene de los clientes (gráfico 4). A pesar de esta opinión, la mayor parte de los empresarios reconoce que su conocimiento sobre la competencia es insuficiente, especialmente aquellos que operan en industrias de alto cambio tecnológico.

Hasta ahora hemos identificado que muchas empresas españolas operan en mercados muy competiti- vos y que, a pesar de no estar familiarizados con el término ic, son conscientes de la importancia de vigilar su entorno. También hemos establecido que su nivel de comprensión de la competencia es bajo.

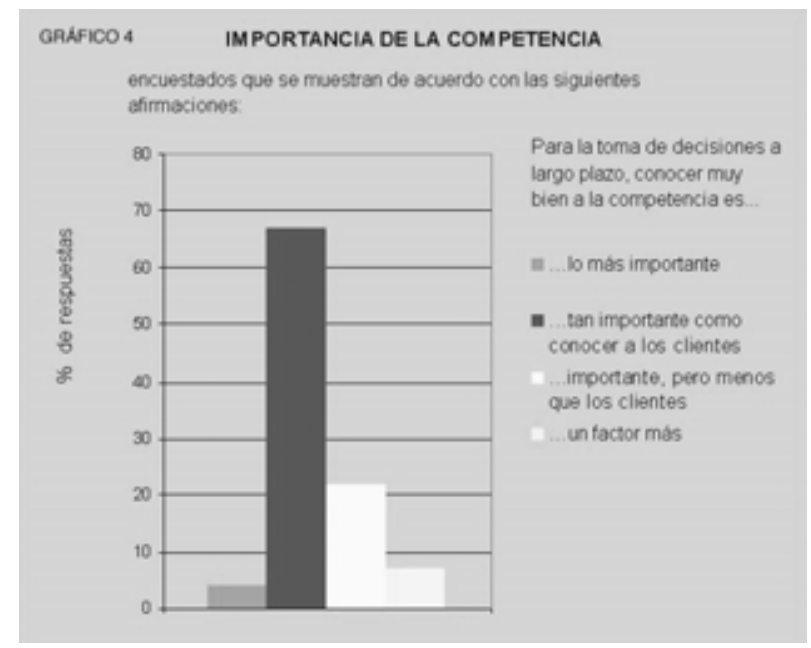

Vamos a analizar ahora los enfoques que adoptan respecto a la ic.

\section{«Las experiencias reales de- muestran que los procesos de ic pueden ser simples y flexi- bles y por lo tanto adaptables tanto a las necesidades como a los recursos de la empresa»}

\section{d. Procedimientos para el análisis del entorno competitivo.}

El 75\% de las compañías analizan el entorno competitivo a través de procedimientos informales o de forma discontinua. Aquellas que utilizan procesos formales de inteligencia declaran tener un conocimiento de sus competidores muy superior a la media (gráficos 5 y 6$)$.

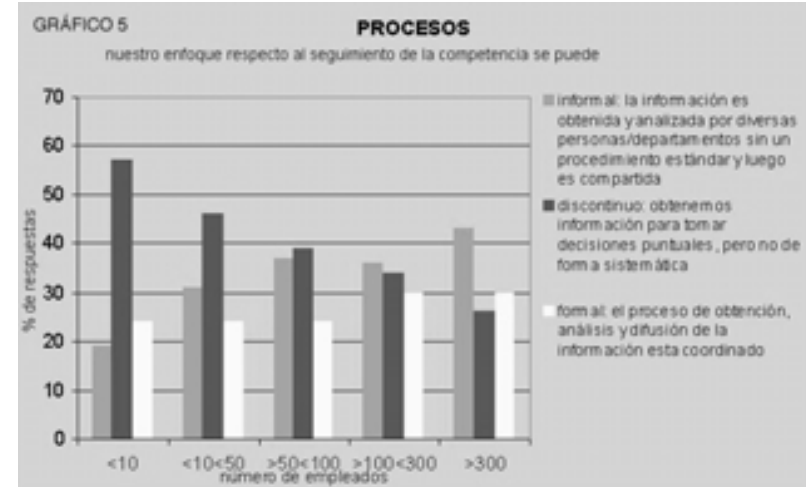

En este informe no se pretende estudiar la posible relación entre utilización formal de ic y éxito de la empresa. Sin embargo, si seguimos las corrientes de pensamiento mayoritarias, y asumiendo que un buen conocimiento de los competidores permite entender e in- 
cluso anticipar sus movimientos, podemos deducir que las empresas que poseen sistemas formales de ic gozan de una clara ventaja respecto de las que no los utilizan, por ser su capacidad de planificación superior.

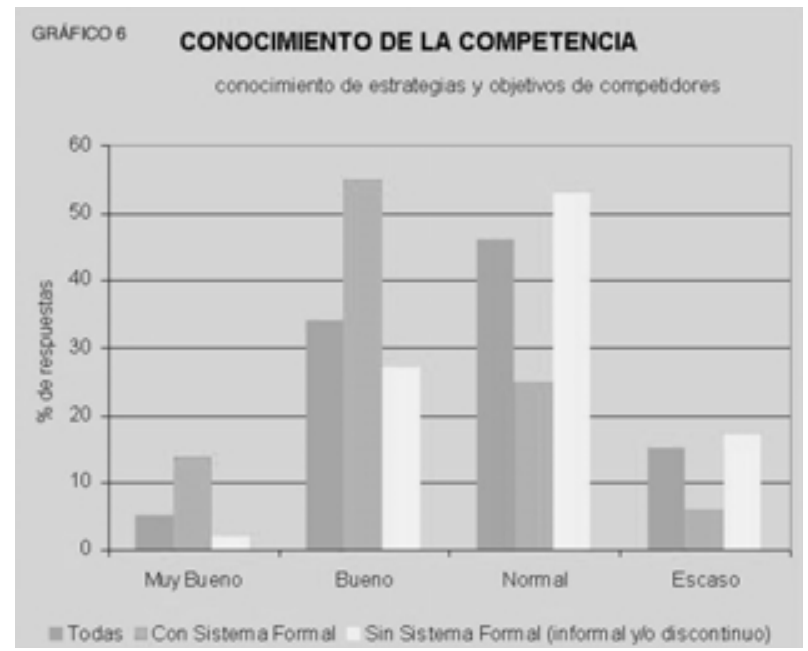

e. Obstáculos para implementar procesos formales.

La cultura de la empresa junto con la falta de experiencia en las técnicas de análisis son las principales razones para no adoptar procesos formales de ic (gráfico 7). Aunque los directivos señalan la falta de recursos humanos como el principal factor que impide su adopción, un análisis detenido permite observar cómo la implementación de estos programas no depende del tamaño de la empresa. Existen casos de pequeñas organizaciones que utilizan con éxito sistemas formales de ic; esto es posible, las experiencias reales demuestran que los procesos de ic pueden ser simples y flexibles y por lo tanto adaptables tanto a las necesidades como a los recursos de la empresa.

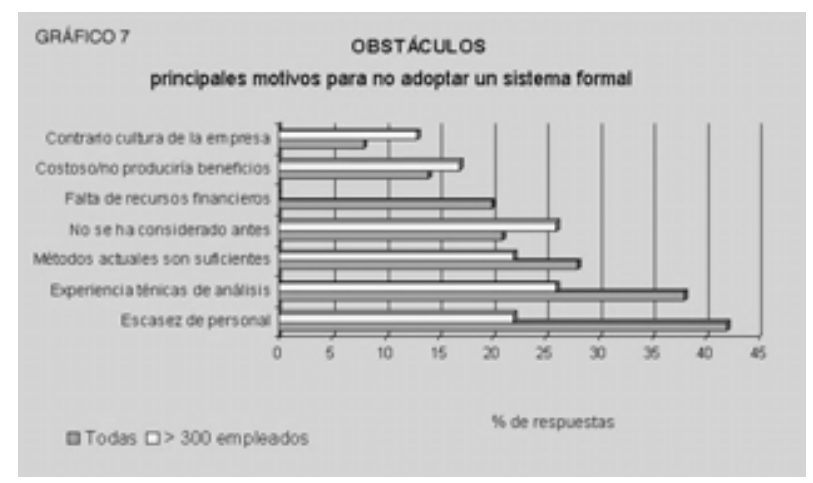

Sin embargo, es la falta de conocimiento en las técnicas de inteligencia el principal obstáculo para la puesta en marcha de un programa formal. Un número significativo de empresarios, a pesar de admitir un escaso conocimiento de su entorno competitivo, no ve la necesidad de implantar sistemas de inteligencia, ya sea porque piensa que sus actuales métodos son suficientes o porque desconoce que haya otros.
Esto nos lleva a suponer que es la cultura interna de la empresa la que determina la existencia de un programa formal de inteligencia. En este área, organismos de promoción de las exportaciones como el Icex (Instituto Español de Comercio Exterior) y las cámaras de comercio, pueden jugar un papel clave dando a conocer entre los exportadores la importancia de estos procesos formales.

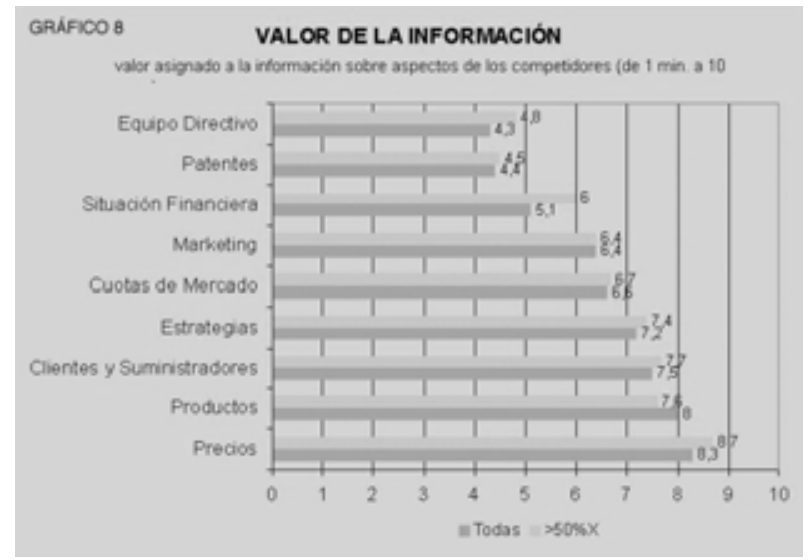

\section{f. El énfasis de la ic.}

La información táctica sobre competidores (precios y productos) es la más valorada por las empresas (gráfico 8). Independientemente del enfoque utilizado (formal, informal o discontinuo), la mayor parte de los directivos conceden mayor valor a la información táctica que a la estratégica. El énfasis en aspectos tácticos significa que en la mayor parte de las sociedades el esfuerzo en inteligencia se centra en lo que los competidores han hecho, y no en lo que pueden hacer en el futuro. Sólo las empresas grandes parecen dar más importancia a la inteligencia con fines estratégicos: desarrollo de producto, relaciones con suministradores, etc. Es de suponer que a medida que tengan acceso a más información táctica de sus rivales (especialmente en internet) sus esfuerzos se concentren hacia cuestiones estratégicas de más largo plazo.

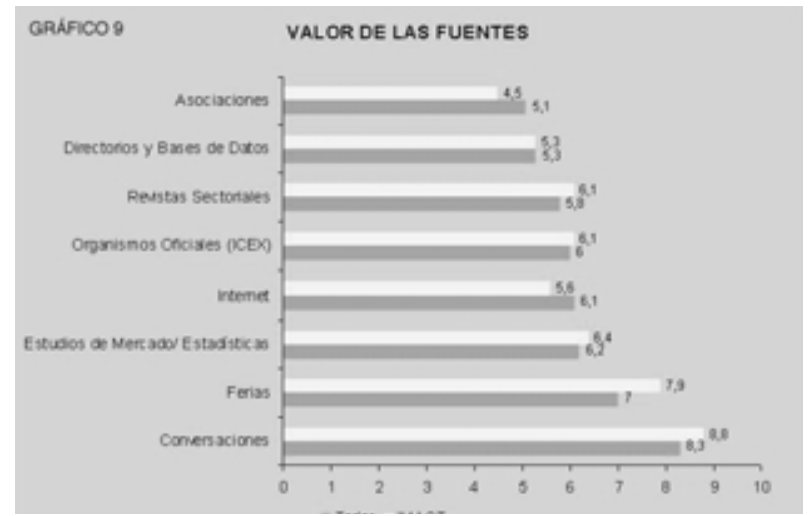

\section{g. El ciclo de la ic.}

Las conversaciones informales son la fuente más valorada de inteligencia (gráfico 9); el 68\% de las em- 
presas no tiene políticas de protección de información interna que sean de aplicación a todos los empleados en general (gráfico 10).

Es de sobra conocido que todo ciclo de inteligencia se compone de al menos cuatro fases:

—obtención de información,

—análisis,

—comunicación de resultados, y

— protección de la propia información.

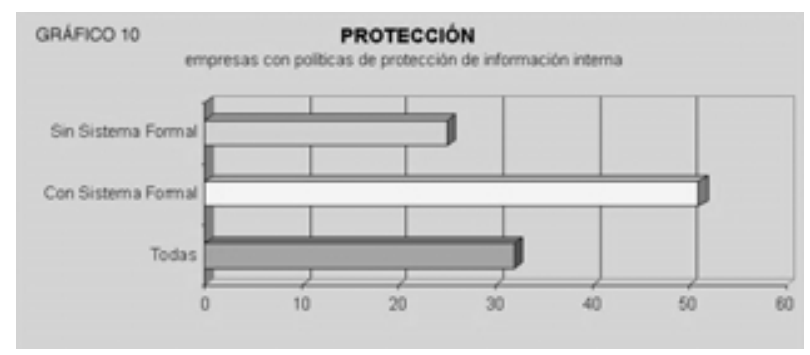

No hay duda de que los ejecutivos españoles hacen buen uso de su red personal de contactos como herramienta de obtención de ic. Sin embargo, una excesiva dependencia de estas fuentes informales puede ser perjudicial si la información está sesgada o es incorrecta. Las experiencias de empresas que utilizan programas de ic demuestran que los más efectivos son aquellos que combinan recursos de información primarios y secundarios, cualitativos y cuantitativos.

\section{«Los programas de ic más efectivos son los que combi- nan recursos de información primarios y secundarios, cuali- tativos y cuantitativos»}

Es significativo que internet no esté especialmente bien valorada como fuente de obtención de información, pero es previsible que su uso irá en aumento a medida que se abaraten los costes de acceso y aumente la velocidad de las líneas. En cambio, el motivo por el cual las instituciones públicas no son uno de los principales recursos sobre la competencia puede obedecer a que la información que buscan las empresas sea específica (generalmente precios de productos competidores) y no suela encontrarse en este tipo de organismos.

La utilización de técnicas de análisis de competidores (dafo - debilidades, amenazas, fortalezas y oportunidades, swot en inglés- escenarios, 5 fuerzas, etc.) depende más de la habilidad y conocimientos de los profesionales que del uso de herramientas sofisticadas. Como hemos sugerido anteriormente, al no requerir inversiones de capital cualquier empresa puede utilizarlas si cuenta con personal entrenado en su uso y aplicación.

Por otra parte, los directivos de empresas con sistemas formales de inteligencia son los que más satisfechos se muestran con la organización interna de la información que necesitan para su labor, es decir, son más eficaces cuando se trata de compartir el conocimiento (gráfico 11). A este respecto algunas compañías han desarrollado fórmulas para fomentar que la información se comparta, generalmente a través de incentivos y facilitando la comunicación de abajo a arriba.

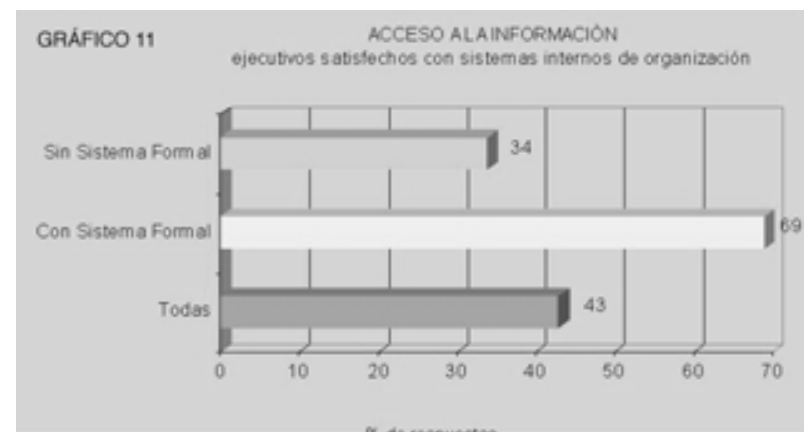

Los resultados de la inteligencia se pueden comunicar de diversas formas, dependiendo de la urgencia e importancia del mensaje: informes cortos o cara a cara para los asuntos más importantes; correos electrónicos y otros medios escritos para cuestiones menos urgentes.

Por último, la protección de la propia información es la fase menos observada del ciclo de inteligencia: pocas empresas toman medidas al respecto. Esta circunstancia es lógica en el caso de compañías sin programas formales de ic, pero es sorprendente en las que estructuran las tres fases anteriores. No olvidemos que una fuga de información confidencial podría dar al traste con el esfuerzo de inteligencia realizado.

\section{Las experiencias de cuatro empresas}

Los datos que a continuación se comentan han sido recogidos a través de organizaciones que operan en mercados dinámicos y competitivos e ilustran cómo se pueden adoptar diferentes sistemas de ic. Debido a que prefieren mantener la confidencialidad utilizaremos nombres supuestos, pero comentando que todas son líderes de sus sectores en España. Dos de ellas son suministradoras de servicios en el mundo de las telecomunicaciones y la energía (de ahora en adelante las denominaremos Telsa y Enesa), mientras que las otras dos son fabricantes de productos de madera y software (Madsa y Sofsa respectivamente).

Las principales diferencias entre sus programas derivan de su orientación, que puede ser táctica o estratégica. Las dos primeras, las más grandes en número de empleados, tienen programas con un enfoque es- 
tratégico dirigido a la alta dirección para la toma de decisiones a largo plazo. Sus unidades de ic están situadas en departamentos de planificación estratégica, y la labor de inteligencia responde a unas necesidades muy concretas, siendo su finalidad suministrar recomendaciones para actuar. Ambas dedican 12 personas a tiempo completo a estas funciones, coordinados por un único responsable, aunque muchas otras personas repartidas por diferentes áreas de la empresa contribuyan a la labor de inteligencia.

«Es significativo que internet no esté especialmente bien valorada como fuente de obtención de información, pero es previsible que su uso irá en aumento»

En cambio, los programas de Madsa y Sofsa son más tácticos, sus unidades están situadas en departamentos de marketing, el producto del esfuerzo de inteligencia es estándar, no está personalizado y no ofrecen recomendaciones al destinatario o cliente final de la inteligencia. En lo que respecta al de Sofsa, presta apoyo a los departamentos de ventas y marketing para tomar decisiones a corto plazo. Para ello 6 personas elaboran y suministran información a cuadros medios y altos de la empresa de forma regular. El de Madsa depende en gran medida de la tecnología, así como de 20 analistas que deben introducir la información en unos soportes informáticos siguiendo unos parámetros definidos.

A pesar de las diferencias sustanciales entre los cuatro programas también se observan similitudes importantes. Por ejemplo, todos ellos se encuentran en constante evolución, están estructurados y todos menos el de Enesa están descentralizados e involucran la participación de más de una división o departamento de la empresa. El elemento humano como recurso fundamental de información está siempre presente, Telsa y Sofsa incluso han ideado sistemas para desarrollar y mantener sus redes de informadores. Al mismo tiempo todos los programas hacen uso de una amplia variedad de fuentes secundarias, principalmente informes de inversores financieros, revistas sectoriales, así como de información localizada en internet.

Del mismo modo, todos los programas de ic analizados cuentan con uno o más "campeones" internos. Los resultados no suelen ser a corto plazo, es una tarea continuada que se extiende en el tiempo. Por este motivo la figura del campeón o líder interno que apueste desde el principio por la labor de inteligencia es crucial. El apoyo por parte de alguien con suficiente peso en la empresa es necesario para garantizar la supervi- vencia de la función, al menos hasta que los resultados le concedan la credibilidad que necesita. Otra semejanza es que todas las organizaciones han desarrollado mecanismos para proteger la información interna confidencial.

\section{«Una fuga de información con- fidencial podría dar al traste con el esfuerzo de inteligencia realizado»}

Las cuestiones éticas en relación con la obtención de inteligencia son también fuente de preocupación, aunque sólo una de las empresas había implementado directrices explícitas al respecto. En cuanto a las principales dificultades para desarrollar su trabajo, las más mencionadas son evitar el exceso de información y conseguir que los clientes de la unidad de ic sigan las recomendaciones que se les hacen.

$\mathrm{El}$ análisis anterior nos invita a realizar tres consideraciones principales:

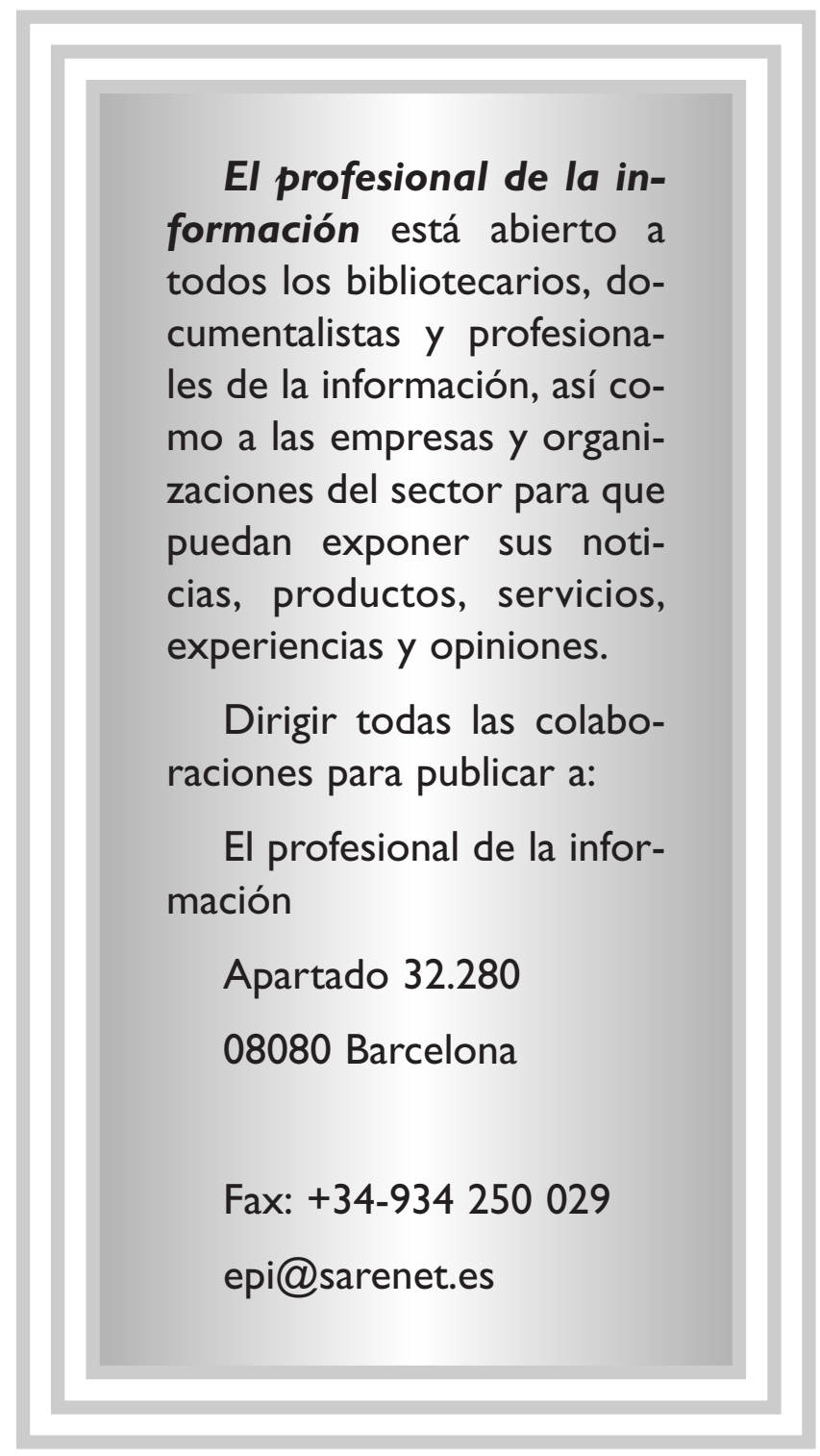


- Los programas de ic suelen estar descentralizados. Parece claro que sólo desconcentrando la función de inteligencia, es decir, inculcando en los empleados la importancia de comunicar a los encargados de inteligencia todo aquello que pueda constituir información valiosa, se logra que los responsables de la unidad, que no pueden ser especialistas en todo, aprovechen al máximo el conocimiento interno de la empresa.

-Parece que la orientación estratégica o táctica de la función de inteligencia dependerá de su localización dentro de la empresa. Las unidades de ic integradas en departamentos de planificación tienen un enfoque estratégico, mientras que las desarrolladas para apoyar actividades de marketing tienen una vertiente más táctica.

- Los mayores retos que los programas de ic deben afrontar son tres: demostrar su contribución a la entidad; establecer un nexo de unión entre recomendaciones de la unidad de inteligencia y toma de decisiones, y evitar el exceso de información.

En realidad sólo se pueden establecer criterios subjetivos para medir los éxitos de un programa de ic. Por este motivo consigue credibilidad sólo si contribuye a conseguir una posición de ventaja competitiva, entendida ésta no sólo en función de beneficios obtenidos sino también de riesgos anticipados y evitados gracias a su uso.

\section{«Las empresas españolas, en especial las pymes, deberán buscar futuras oportunidades en el extranjero para poder continuar compitiendo en mer- cados cada vez más globales y dinámicos»}

Para ello es necesario que los analistas de ic sean capaces de convencer a los directivos de las ventajas de utilizar sus recomendaciones en la toma de decisiones. Conseguir y fortalecer esta relación entre análisis y decisión es clave. Para facilitarlo algunas empresas sitúan a dichos analistas cerca de la alta dirección, esperando que sus dotes de persuasión y comunicación hagan el resto.

En cuanto al exceso de información, la forma más efectiva de evitarlo es trabajar con unos objetivos específicos y respondiendo a necesidades concretas de clientes internos que deben estar claramente identificadas. Los programas demasiado genéricos que se limitan a difundir información sin priorizar consiguen que sus abrumados clientes pierdan el interés y terminan por fracasar.

\section{Conclusiones y recomendaciones}

Los directivos españoles coinciden en señalar que un buen entendimiento del entorno competitivo es fundamental para tomar decisiones y que las empresas españolas, en especial las pymes, deberán buscar futuras oportunidades en el extranjero para poder continuar compitiendo en mercados cada vez más globales y dinámicos. En definitiva, será necesario tomar decisiones para aumentar sus exportaciones y presencia en el exterior.

A este respecto, los resultados de la investigación confirman las hipótesis iniciales:

- La existencia de sistemas formales de obtención, análisis y difusión de información concede una ventaja competitiva a las empresas, ya que facilita a la dirección una mejor visión del entorno y de los factores que inciden en su negocio. Así, las que implementan sistemas formales de ic son capaces de comprender, y en ocasiones de predecir, los movimientos de sus competidores. Cuando estos conocimientos son utilizados en los procesos internos de decisión el resultado es que la estrategia elegida se ve fortalecida.

\section{«El estudio indica que, por lo general, los exportadores espa- ñoles tienen un conocimiento mediocre de sus rivales»}

- Todas las compañías, independientemente de su envergadura, se pueden beneficiar de programas formales de ic. El tamaño condiciona la existencia de sistemas discontinuos o informales, pero no es determinante en su adopción. A este respecto, la cultura interna de la empresa y el conocimiento de las técnicas de análisis tienen una gran influencia. Debido a que los programas formales pueden tener diferentes niveles de complejidad, es necesario que se adapten a las propias necesidades y estructuras.

El estudio indica que por lo general los exportadores españoles tienen un conocimiento mediocre de sus rivales. La principal causa de esta ignorancia parece ser, precisamente, la falta de sistemas formales de inteligencia. El 75\% de las empresas sondeadas no los utilizan y al parecer no saben cómo obtener, analizar y compartir información sobre su entorno de una manera eficaz.

- Las experiencias reales de cuatro organizaciones han recogido los aspectos más importantes de los procesos de inteligencia utilizados en distintos entornos. Las funciones o unidades de ic analizadas tienen una orientación táctica o estratégica según su localización dentro del organigrama interno. Si está integrada, o sirve de apoyo, a departamentos de ventas o marke- 
ting el énfasis de su función es hacia aspectos tácticos o de corto plazo. Si se sitúa cercana a la dirección su carácter es más estratégico o a largo plazo.

No existe un modelo único de ic. Los programas analizados poseen algunas de las características que se suelen considerar como más efectivas, aunque no todas. Las particularidades de cada uno dependen de su nivel de evolución, de las necesidades de la empresa y del valor que otorgue la dirección a la función de inteligencia, pero no implican necesariamente la implementación de procesos complejos o costosos.

En general se considera que para mejorar las posibilidades de éxito de los programas y/o unidades formales de ic es necesario que:

—cuenten con el apoyo de la dirección,

—impliquen a empleados repartidos por diversas áreas de la empresa,

- tengan una fuerte orientación hacia el trabajo por encargo y no a la difusión de información de forma indiscriminada, y

—evolucionen para adaptarse a nuevas necesidades.

Estas características ayudarán a superar los principales retos que toda función de inteligencia deberá afrontar: la medición y justificación de su efectividad y productividad; conseguir que la dirección tenga en cuenta sus recomendaciones en la toma de decisiones y evitar el exceso de información.

\section{Bibliografía}

Amboise, G.; D’Muldowny, M. "Management theory for small businesses: attempts and requirements". En: Academy of management review, 1988 , v. 13 , n. 2 , pp. 226-240.

Bamberg, I. "Value systems, strategies and the performance of the SMEs". En: European small business journal, 1:4, pp. 25-39.
Brouthers, K.; Andriessen, F.; Nicolaes, I. "Driving blind: strategy decision making in small companies". En: Long range planning, 1998, v. 31, n. 1, pp. 130-138.

Calof, J. "Opportunity intelligence: the missing ci tribe". En: Competitive intelligence magazine, 2000, julio-septiembre, v. 3, p. 35.

Cottrill, K. "Turning competitive intelligence into business knowledge". En: Journal of business strategy, 1998, julio-agosto, v. 19, n. 4, p. 27.

Fuld, L. The new competitor intelligence. The complete guide for finding, analysing, and using information about your competitors. Chichester: John Wiley \& Sons, Inc., 1994. 512 p. Isbn: 0471585092.

Giménez Toledo, E.; Román Román, A. "Vigilancia tecnológica e inteligencia competitiva: conceptos, profesionales, servicios y fuentes de información". En: El profesional de la información, 2001, v. 10, n. 5, mayo, pp. 11-20.

Henricks, M. "Spy away (competitive intelligence for small companies)". En: Entrepreneur, 2000, marzo, v. 28, n. 3, p. 98.

Hussey, D.; Jenster, P. Competitor intelligence: turning analysis into success. Chichester: John Wiley \& Sons, Inc., 1999. Isbn: 0471984078.

Orozco Silva, E. "La inteligencia empresarial en la gestión del conocimiento". En: El profesional de la información, 2001, v. 10, n. 7-8, julioagosto, pp. 14-22.

Palop, F.; Vicente, J. M. Vigilancia tecnológica e inteligencia competitiva: su potencial para la empresa española. Fundación Cotec.

Pawar, B. S.; Sharda, R. "Obtaining intelligence on the internet". En: Long range planning, 1997, v. 30, n. 1, pp. 110-121.

Porter, M. "What is strategy?". En: Harvard business review, 1996, noviembre-diciembre.

Prescott, J.; Gibbons, P. "The seven seas of global competitive intelligence". En: Competitive intelligence review, v. 7, supplement n. 1, S41S48.

Tena Millán, J.; Comai, A. "Los propósitos de la inteligencia en la empresa: competidora, cooperativa, neutral e individual". En: El profesional de la información, 2001, v. 10, n. 5, mayo, pp. 4-10.

\section{Próximos números monográficos}

Diciembre 2001 Especial $10^{\circ}$ aniversario EPI

Enero 2002 Intranet y documentación

Marzo 2002 Automatización de bibliotecas

Los interesados pueden remitir notas, artículos, propuestas, publicidad, comentarios, etc., sobre estos temas a:

epi@sarenet.es 